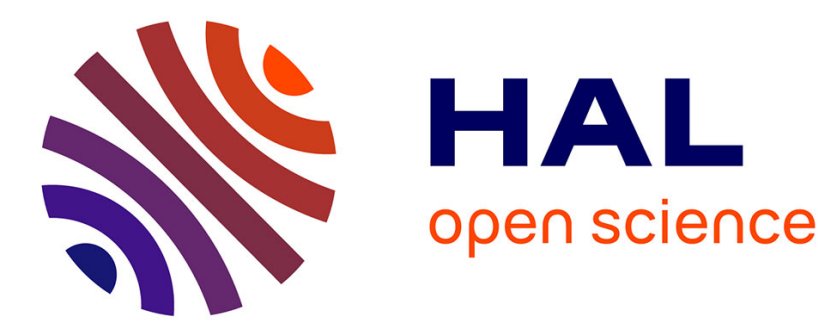

\title{
Copper(I)-mediated protein-protein interactions result from suboptimal interaction surfaces
}

\author{
Lucia Banci, Ivano Bertini, Vito Calderone, Nunzia Della-Malva, Isabella \\ Caterina Felli, Sara Neri, Anna Pavelkova, Antonio Rosato
}

\section{- To cite this version: \\ Lucia Banci, Ivano Bertini, Vito Calderone, Nunzia Della-Malva, Isabella Caterina Felli, et al.. Copper(I)-mediated protein-protein interactions result from suboptimal interaction surfaces. Bio- chemical Journal, 2009, 422 (1), pp.37-42. 10.1042/BJ20090422 . hal-00479175}

\section{HAL Id: hal-00479175 \\ https://hal.science/hal-00479175}

Submitted on 30 Apr 2010

HAL is a multi-disciplinary open access archive for the deposit and dissemination of scientific research documents, whether they are published or not. The documents may come from teaching and research institutions in France or abroad, or from public or private research centers.
L'archive ouverte pluridisciplinaire HAL, est destinée au dépôt et à la diffusion de documents scientifiques de niveau recherche, publiés ou non, émanant des établissements d'enseignement et de recherche français ou étrangers, des laboratoires publics ou privés. 


\title{
Copper(I)-mediated protein-protein interactions result from suboptimal interaction surfaces
}

Lucia Banci ${ }^{*, \dagger,}$, Ivano Bertini ${ }^{*, \dagger}$, Vito Calderone ${ }^{\star}$, Nunzia Della-Malva*, ${ }^{\star, \dagger}$ Isabella C. Felli*, ${ }^{*,}$, Sara Neri* $^{\star}$, Anna Pavelkova* ${ }^{\star}$ Antonio Rosato ${ }^{\star \dagger}$

* Magnetic Resonance Center (CERM) - University of Florence, Via L. Sacconi 6, 50019 Sesto Fiorentino, Italy

${ }^{\dagger}$ Department of Chemistry - University of Florence, Via della Lastruccia 3, 50019 Sesto Fiorentino, Italy

${ }^{\ddagger}$ FIORGEN Foundation, Via L. Sacconi 6, 50019 Sesto Fiorentino, Italy

Short title: Interaction surfaces of metal-mediated protein adducts

\author{
Corresponding Author: \\ Prof. Ivano Bertini \\ Magnetic Resonance Center \\ University of Florence \\ Via L. Sacconi, 6 \\ 50019 Sesto Fiorentino, ITALY \\ Fax: +390554574271 \\ Tel: +390554574272 \\ E-mail: ivanobertini@cerm.unifi.it
}

Keywords: HAH1; ATP7A; ATP7B; Menkes disease; metal homeostasis

\footnotetext{
Abbreviations: MNK $n: n^{\text {th }}$ metal-binding domain of the Menkes disease protein ATP7A; WLN $n$ : $n^{\text {th }}$ metal-binding domain of the Wilson disease protein ATP7B; DTT: dithiothreitol
} 
Interaction surfaces of metal-mediated protein adducts

\section{Synopsis}

The homeostasis of metal ions in cells is the result of the contribution of several cellular pathways that involve transient, often weak, protein-protein interactions. Metal transfer typically implies the formation of adducts where the metal itself acts as a bridge between proteins, by coordinating residues of both interacting partners. Here we addressed the interaction between the human copper(I)-chaperone HAH1 and one metal-binding domain of one of its partners, namely the P-type copper-transporting ATPase ATP7A. The adduct was structurally characterized in solution, in the presence of copper(I), and through X-ray crystallography, upon replacing copper(I) with cadmium(II). Further insight was obtained through molecular modelling techniques and sitedirected mutagenesis. It is found that the interaction involves a relatively small interface (less than $1000 \AA^{2}$ ) with a low fraction of non-polar atoms. These observations provide a possible explanation for the low affinity of the two apo-proteins. It appears that electrostatics is important in selecting which domain of the ATPase is able to form detectable amounts of the metal-mediatd adduct with HAH1.

\section{Introduction}

Many functional processes in the cell occur through weak, transient interactions among proteins. For these cases, the interaction is associated to a small, negative change in free energy. Indeed, if the free energy change were large and negative, the complex would be stable and nontransient, whereas if it were positive, no interaction would occur. The study of these interactions is crucial for the thorough understanding of the mechanisms at the basis of Life and of their alterations caused by mutations. Metal homeostasis in cells, which includes uptake, controlled distribution to metalloproteins, storage, and excretion of metal ions, is a process involving many transient interactions.

Copper is utilized as a cofactor in a variety of proteins [1], but it can be potentially toxic in vivo and thus its intracellular concentration is presumably strictly controlled [2-4]. Its traffic and homeostasis occur through copper-mediated protein-protein interactions [5], where copper(I) is bound to ligands from both proteins of the complex. The free energy of formation of copper(I)mediated protein-protein adducts is the outcome of the balance of the metal-donor(s) bond energies, of the hydrophobic and hydrophilic interaction energies at the interface and of the entropic implications related to the establishment of the above interactions.

In order to better understand the processes of metal binding and transfer, we have focused on copper trafficking from the cytoplasm to the trans-Golgi network (TGN), where copper is inserted in a number of enzymes [6]. In the cytoplasm, copper(I) is bound to a metallochaperone which delivers the metal to a variable number of soluble metal-binding domains (MBDs) of specific ATPases located at the TGN membrane [2]. In yeast, the metallochaperone is called Atx1 and the ATPase is Ccc2 [7]. In humans, the metallochaperone is called Atox 1 or HAH1 and two target ATPases exist: ATP7A and ATP7B [8]. Both ATP7A and ATP7B have six soluble MBDs. The present research aims at shedding light on the nature of the interaction between the metallochaperone and a MBD of the ATPases in the general frame of understanding the energetics of copper(I)-mediated interactions. With this in mind, we addressed the study of the copper(I)mediated complex between HAH1 and the first MBD of ATP7A (MNK1). We also investigated whether introducing cadmium(II) instead of copper(I) had an impact on the interaction. The structures of the copper(I)- and cadmium(II)-mediated adducts are here reported, the former in solution and the latter as a crystal (whose existence in solution was validated by ${ }^{113} \mathrm{Cd} \mathrm{NMR}$ ). We probed some of the key interactions observed in the structures by site-directed mutagenesis. 


\section{Experimental}

\section{Sample preparation}

We cloned and expressed the first soluble metal-binding domain of ATP7A (MNK1), corresponding to amino acids 5-77 of the full-length protein, using essentially the same protocol adopted for other individual MNK domains [9]. WT- and mutant HAH1 protein samples were produced as previously reported [10]. Further details are supplied in the Supplementary Information section.

\section{Structural analysis}

All NMR experiments were collected at 298K except on Bruker Avance spectrometers equipped with a triple resonance cryoprobe. The experiments for the sequence-specific assignment of resonances were acquired on the samples in which only one of the partners were ${ }^{13} \mathrm{C},{ }^{15} \mathrm{~N}$ labelled, while the other was unlabeled. The $1 \mathrm{D}{ }^{113} \mathrm{Cd}$ experiment was acquired with a BBO probe at 600 MHz. Tables S1, S2 report details on the NMR experiments performed. The dynamics of the protein backbone was investigated through ${ }^{15} \mathrm{~N}$ relaxation [11] and allowed us to estimate the overall rotational correlation time of the molecules (Table S3). Combined NMR chemical shift variations were calculated from the experimental ${ }^{1} \mathrm{H}$ and ${ }^{15} \mathrm{~N}$ chemical shift variations $\left(\Delta \delta\left({ }^{1} \mathrm{H}\right)\right.$ and $\Delta \delta\left({ }^{15} \mathrm{~N}\right)$, respectively) measured between corresponding peaks, through the following equation [12].

$$
\Delta \delta^{\text {combined }}=\sqrt{\frac{\left(\Delta \delta\left({ }^{1} \mathrm{H}\right)\right)^{2}+\frac{1}{25}\left(\Delta \delta\left({ }^{15} \mathrm{~N}\right)\right)^{2}}{2}}
$$

The intramolecular cross-peaks used for structure calculations were extracted from 3D NOESYHSQC spectra acquired on samples where the apo-form of one of the partners, enriched in ${ }^{15} \mathrm{~N}$ and ${ }^{13} \mathrm{C}$, was mixed with a 1.5 molar excess of the unlabeled copper(I)-form of the other partner. The intermolecular cross-peaks were integrated in $\omega_{1-}{ }^{13} \mathrm{C}$-edited, $\omega_{2-}{ }^{13} \mathrm{C}$-separated, $\omega_{3}{ }^{-1}{ }^{13} \mathrm{C}$, ${ }^{15} \mathrm{~N}$-filtered NOESY experiments acquired on samples containing 1:1:1 mixture of copper(I):enriched partner:unlabeled partner.

Crystals of HAH1-Cd(II)-MNK1 belonging to the spacegroup $\mathrm{P} 2{ }_{1}{ }_{2}{ }_{1}{ }_{1}$ were obtained using the sitting drop vapor diffusion method at $16^{\circ} \mathrm{C}$ from a solution containing $0.1 \mathrm{M}$ sodium citrate, $20 \%$ PEG-6000, 0.8mM complex concentration, at pH 4.7.

Structural models of all MBDs of the MNK protein were built using the program Modeller v9.5 [13], and then used in protein-protein docking calculations with the program HADDOCK [14].

The experimental structures of HAH1-Cu(I)-MNK1 and HAH1-Cd(II)-MNK1 are available from the PDB ( $2 \mathrm{~K} 1 \mathrm{R}$, including chemical shift data, and 3CJK, respectively). The coordinates of the modelled MBD:HAH1 structures are available from http://www.cerm.unifi.it/modstruc/MNKdomains_HAH1modeladducts.zip. Further details on structure determination and analysis are given in the Supplementary Information.

\section{Results and Discussion}

\section{Formation of the HAH1-Cu(I)-MNK1 and HAH1-Cd(II)-MNK1 adducts}

An equimolar mixture of HAH1 and of the first domain of ATP7A (MNK1) formed a metalmediated adduct with 1:1:1 stoichiometry when presented with $\mathrm{Cu}^{+}$. The complex was in fast (on the NMR timescale) exchange with the free proteins. A similar adduct is presumably formed by 
HAH1 and MNK4 [15;16], whereas this does not happen with domains 2, 5, 6 of ATP7A [17;18]. By measuring the reorientation time for molecular tumbling in solution of MNK1 (5.5 ns) and of HAH1 (4.6 ns), and comparing it to the corresponding value for the complex ( $8.9 \mathrm{~ns})$, it can be deducted that ca. $88 \%$ of the protein is involved in the intermolecular complex. In addition, it is known that the relative affinity for copper(I) of the various MBDs of ATP7A is similar and approximately ten times that of HAH1 [17-19]. From these data, it can be estimated that the constant for the equilibrium is close to $10^{5} \mathrm{M}^{-1}$.

We additionally investigated the interaction of the HAH1:MNK1 mixture, prepared in the absence of metal ions, with $\mathrm{Zn}^{2+}$ and $\mathrm{Cd}^{2+}$. Addition of $\mathrm{Zn}^{2+}$ led to extensive sample precipitation, whereas $\mathrm{Cd}^{2+}$ induced formation of an adduct at essentially $100 \%$ completion, as shown by the HSQC spectra of both partners in the mixture. $1 \mathrm{D}^{113} \mathrm{Cd}$ NMR spectra were recorded to obtain hints on the chemical identity and number of the donor atoms. A shift of $680 \mathrm{ppm}$ was measured, which is consistent [20] with the ${ }^{113} \mathrm{Cd}$ environment seen in the crystal structure. The adduct was in slow rather than fast equilibrium with the free proteins. HAH1 and MNK6 are known not to form an adduct when mixed at 1:1 ratio in the presence of equimolar copper(I) [17]. Also with $\mathrm{Cd}^{2+}$, an adduct between HAH1 and MNK6 could not be detected. In addition, we did not observe the formation of $\mathrm{Cu}^{+}$or $\mathrm{Cd}^{2+}$-mediated HAH1 dimers, which have been reported under different experimental conditions $[21 ; 22]$.

\section{The structures of HAHI and MNK1 within the HAH1-Cu(I)-MNK1 adduct}

The structure of the HAH1-Cu(I)-MNK1 complex was solved using a combination of: i) intramolecular NOEs and dihedral angle restraints measured on protein samples containing the apoform of one of the two partners enriched in ${ }^{15} \mathrm{~N},{ }^{13} \mathrm{C}$ in the presence of a 1.5 molar excess of the copper(I)-form of the unlabeled partner; ii) intermolecular NOEs measured on protein samples containing the same proteins as for i) but at a 1:1 ratio; iii) distance restraints between the $\mathrm{S} \gamma$ atoms of the cysteines and the copper(I) ion based on the results of mutagenesis experiments (see section on metal coordination). The data from i) allowed us to obtain the solution structure of each protein when $100 \%$ in the complex, whereas the distance restraints from ii) originate solely from the complex. This procedure allowed us to confidently assess the structural rearrangements due to complex formation, which has not been possible for the Atx1-Cu(I)-Ccc2a adduct [5]. The structure of the HAH1-Cd(II)-MNK1 complex was solved at $1.8 \AA$. Statistics for the two structures are given in Tables S4 and S5, respectively.

The solution structure of copper(I)-HAH1 (PDB entry 1TL4) [10] could be superimposed very well to the present structure of HAH1 in the HAH1-Cu(I)-MNK1 adduct (Figure 1), with a backbone RMSD between the two mean structures of $0.95 \AA$ (Table S6). Significant differences could be noted at the end of the first $\beta$ strand for residues 8-10, located before the metal-binding loop and for the region of loop $\mathrm{V}$, encompassing residues 57-60. Other changes regarded the orientation of side chains at the protein-protein interface, e.g. the side chain of Arg21 experienced a significant rearrangement to accommodate the partner. In HAH1 alone, the side chain of Lys60 rearranges upon metallation, pointing toward the loaded metal site [10]; in the present structures, this side chain points towards the partner molecule, with a conformation similar to that observed in apo-HAH1. The NMR structure of copper(I)-MNK1 (PDB entry 1KVJ) [23] had a backbone RMSD to the present one of $1.4 \AA$ (Table S7). Note that $1 \mathrm{KVJ}$ had five amino acids at the Nterminus more than the present one and was solved under different experimental conditions. Significant differences were observed for the backbone conformation of loops I and V; in addition, a reorientation of helix $\alpha_{1}$ was apparent. A rearrangement of the same helix has been reported for yeast Ccc2a upon interaction with its partner [5].

The structures of HAH1-Cu(I)-MNK1 and HAH1-Cd(II)-MNK1 
Figures 1 and 2 show respectively the structures of HAH1-Cu(I)-MNK1 and HAH1-Cd(II)MNK1 determined in this work. The two structures were similar, with the HAH1-Cu(I)-MNK1 interface being smaller and slightly rotated with respect to HAH1-Cd(II)-MNK1. A list of contacts across the interface for the two complexes is given in Table 1. Most contacts, except those in the metal-binding loop I, are conserved in both structures.

Table 2 reports an analysis of the protein-protein interface for HAH1-Cd(II)-MNK1, HAH1$\mathrm{Cu}(\mathrm{I})-\mathrm{MNK} 1$ (shown in Figure 3), yeast Atx1-Cu(I)-Ccc2a, and $\mathrm{Cd}-\mathrm{HAH} 1_{2}$. Some consistent features are observable: i) the total interface area is between 800 and $1000 \AA^{2}$, i.e. $400-500 \AA^{2}$ per partner, covering slightly more than $10 \%$ of the total surface; ii) the fraction of non-polar atoms at the interface is, on average, between $54 \%$ and $64 \%$; iii) the propensity score of the interfaces is negative. The propensity score is calculated on the basis of the residue composition of the interfaces, using the pre-computed propensity of each amino acid [24]. The latter represents a measure of how much more (positive propensity) or less (negative propensity) frequently a given amino acid is at a protein-protein interface than just on the protein surface. The interface areas were smaller than "standard" areas for protein-protein complexes, which are typically at least $1000 \AA^{2}$ [24;25]. However, because these are relatively small proteins, the interface/total area ratio is within the lower end of the distribution of values from complexes in the PDB. The fraction of non-polar atoms at the interface was also relatively low. The negative propensity score indicates that the interfaces analysed here were composed mainly by residues that, when at the protein surface, are more likely not to be at the interaction interface. This is due to an over-representation of Gly, Ala and charged residues at the interface, in particular in HAH1 (Table 2). For example in HAH1Cd(II)-MNK1 the core of the interface comprised Cys12, Gly13, Lys57, Thr58, Gly59 of HAH1, all of which have negative propensities, and Met65, Gly66, Phe67 and Asp68 of MNK1, of which only Met and Phe are favoured at interfaces. Cumulatively, these data provide a consistent, general description of how the metallochaperone/ATPase pair(s) is designed to prevent the formation of stable adducts in the absence of the metal ion.

We then performed molecular modelling followed by protein-protein flexible docking to create models of the structures of the adducts of all six MBDs of ATP7A with HAH1. We did not include the metal explicitly, as we wanted to evaluate the different energetic properties of the protein-protein interface under the assumption of a constant contribution from copper(I) coordination. Table 3 shows a comparison of the calculated energies of interaction at the end of the docking simulation. Note that to enable a direct comparison, also the structure of the MNK1-Cu(I)HAH1 was recalculated with the same strategy. The cumulative HADDOCK scores identified the first, fourth and fifth MBDs as those for which the interaction should be most favourable. Of these three, only for the first and fourth MBDs the convergence rate [14] was greater than 50\%. When looking at the calculated energies, it appeared that the largest significant difference among all the complexes formed by the six MBDs was in the predicted electrostatic energy of interaction, which was more favourable for the first and fourth MBDs by 150 to $300 \mathrm{kcal} \mathrm{mol}^{-1}$ (Table 3). The differences among other energetic terms were within 10-20 kcal mol ${ }^{-1}$. This constitutes a demonstration of the key role of electrostatics in domain selection.

We further characterized the MNK1-Cu(I)-HAH1 complex by mutating some amino acids of HAH1 at the protein-protein interface. Arg21, Val22 and Thr58 of HAH1 were targeted. Arg21 is conserved or conservatively substituted by Lys in eukaryotic metallochaperones [26], and is involved in an intermolecular salt bridge with Asp68 of MNK1 (Table 1). A negatively charged residue is commonly, but not strictly, found at position 68 of eukaryotic MBDs. We mutated Arg21 to Gln in order to remove its positive charge. Val22 is present or is conservatively replaced by Ile or Leu in the large majority of all metallochaperones. It is replaced by Ala in the homologue protein from C. elegans. The side chain of Val22 points towards the partner and makes contacts with the backbone of amino acids 64-66 of MNK1. We mutated Val22 to Ala. Finally, Thr58 is fairly conserved in eukaryotic metallochaperones. Its side chain makes hydrogen bonds with the backbone of Leu55, whereas the backbone atoms are involved in intermolecular contacts. We mutated Thr58 
to Ala. All three mutants could bind copper(I) and were capable of interacting with MNK1. The mutations of Arg21 and Val22 had little impact on the structure, as shown by the high similarity of the HSQC spectra of the WT and mutant proteins. The strength of the interaction for the WT and mutant HAH1 systems could be straightforwardly evaluated by analysing the combined chemical shift variations obtained at the same HAH1 and MNK1 concentrations (Figure 4). Both the Arg21Gln and Val22Ala mutants featured a weaker interaction with the partner; the decrease of the combined chemical shift variations was similar for the two mutants. For Arg21Gin, this effect can be ascribed to the loss of the electrostatic interaction. In the case of Val22Ala, the reduced interaction can be ascribed to the loss of the favourable contribution due to the desolvation of the hydrophobic side chain of Val22 upon complex formation. Instead, the HSQC spectrum of the Thr58Ala mutant was significantly perturbed, and showed the occurrence of multiple protein conformations. In addition, several peaks were broadened beyond detection at the first addition of MNK1. Despite these complications, it appeared that the combined chemical shift variations upon interaction with $\mathrm{Cu}(\mathrm{I})-\mathrm{HAH} 1$ were as large as that of the wild-type protein, but the maxima did not correspond to the same amino acids (Figure 4). The HAH1-Cu(I)-MNK1 adduct was thus formed by the Thr58Ala mutant to an extent similar to that of the WT protein, but possibly with a distorted conformation.

\section{Metal coordination}

We used site-directed mutagenesis to investigate the role of individual cysteines in the formation of the HAH1-Cu(I)-MNK1 adducts. To this end, we observed through HSQC spectra whether the metal-mediated adduct was formed upon addition of the metal ion (at equimolar ratio) to a 1:1 mixture containing the wild-type (WT) form of one protein and a mutant of the other protein lacking one of the two copper(I)-binding cysteines. Only three cysteines were needed for the formation of the HAH1-Cu(I)-MNK1 adduct. The two cysteines of each partner that are more Nterminal in sequence (Cys12 of HAH1 and Cys15 of MNK1) were strictly required for the interaction, whereas the third cysteine could be either Cys 15 of HAH1 or Cys 18 of MNK1.

The crystal structure of HAH1-Cd(II)-MNK1 (determined at $\mathrm{pH} 4.7$ ) provides independent information on $\mathrm{Cd}^{2+}$ coordination. The metal ion was tetra-coordinated by the side chains of the two cysteines of MNK1 and of Cys12 of HAH1. The fourth ligand was provided by the hydroxyl of Thr14 of MNK1, leading to an elongated tetrahedral geometry. Thus, MNK1 provided three ligands and HAH1 only one. The $\mathrm{Cd}^{2+}-\mathrm{O} \gamma-\mathrm{C} \beta$ angle was about $150^{\circ}$, suggesting that the hydroxyl group may be deprotonated. Site-directed mutagenesis showed that both cysteines of MNK1 were necessary for the formation of the complex. In the $\mathrm{Cd}-\mathrm{HAH} 1_{2}$ structure [22], the $\mathrm{Cd}^{2+}$ ion is instead at the center of an essentially regular tetrahedron formed by the four $S \gamma$ atoms of the metal-binding cysteines.

In both adducts, the net charge at the metal site is -2 . Instead, the charge at the more solventexposed site in isolated $\mathrm{Cu}(\mathrm{I})-\mathrm{HAH} 1$ is -1. In addition, upon complex formation the distance from the metal site of the $\mathrm{N} \varepsilon$ of Lys60 of HAH1 increased. These effects could be compensated by the favourable orientation of the dipole moment of the first helix of the incoming MNK1 molecule.

\section{Concluding remarks}

The whole body of the data on the interaction between metallochaperones and their partner ATPases, as well as studies with copper(I) chelators [27], demonstrate that metal homeostasis in cells involves a subtle balance between the control of metal reactivity and the capability to transfer the metal between proteins with an appropriate kinetics. The use of metallochaperones to deliver copper to specific intracellular targets has been likened to the action of an enzyme lowering the activation barrier for copper transfer to their specific partners [28]. Additionally, the fine tuning of the identity of the amino acids at interface may allow the metallochaperone-based system to select among different metal ions. The selection can result from the complete abrogation of the formation of the metal-mediated adduct or from a kinetic trap in which the adduct gets locked [29]. The 
present mutagenesis data indicate that it is not a single amino acidic substitution that plays a decisive role. Instead, the network of Van der Waals and electrostatic interactions taking place among the interface residues as well as, presumably, the variation of the conformational space available to the same residues determine the configuration of the adduct and its energy of formation. Structural modelling suggested that for the various MBDs of ATP7A, the electrostatic contribution is crucial to determine the energetics of the interaction with the metallochaperone.

Protein recognition takes place only in the presence of the copper(I) ion, as a result of the small interface area and low propensity of the surface of HAH1 for forming a stable intermolecular complex. This, together with the fact that the affinity for copper(I) of the MBDs of ATP7A is approximately a ten-fold higher than that of HAH1 [17-19], suggests that upon interaction the metal ion is effectively transferred to the ATPase. However, for some MBDs within the soluble tail, such as MNK1, an intermolecular adduct is formed at detectable levels, involving one cysteine residue from HAH1. While the latter can be regarded as a weakly trapped intermediate along the metaltrafficking pathway, its formation might also be instrumental to determine rearrangements of the inter-domain interactions of the ATPase. The latter inter-domain interactions and their variations during the catalytic cycle are important for the enzymatic function of the protein [30;31], as well as to determine the intracellular localization of the ATPase [32]. The formation of adducts between HAH1 and the first or fourth MBD of ATP7A can thus modulate the interaction of the N-terminal cytosolic tail with the other protein domains, ultimately constituting a means for the cell to modulate enzyme turnover [33].

\section{Funding}

Financial support from MIUR (project PRIN 2005), from the EC (contract 031220, SPINE2COMPLEXES) and from the Ente Cassa di Risparmio di Firenze (Projects "Basi Molecolari di patologie umane correlate a disfunzioni della catena respiratoria" and "Relazione varianti proteiche strutturali-malattie genetiche") is acknowledged. The HADDOCK web server is supported by the EC through the eNMR project (contract 213010).

\section{Suppelementary Information Available:}

Supplementary Table S1: Acquisition parameters for NMR experiments performed on HAH1$\mathrm{Cu}(\mathrm{I})-\mathrm{MNK} 1$ containing ${ }^{13} \mathrm{C},{ }^{15} \mathrm{~N}-\mathrm{MNK} 1$

Supplementary Table S2: Acquisition parameters for NMR experiments performed on HAH1$\mathrm{Cu}(\mathrm{I})-\mathrm{MNK} 1$ containing ${ }^{13} \mathrm{C},{ }^{15} \mathrm{~N}-\mathrm{HAH} 1$

Supplementary Table S3: Acquisition parameters for NMR experiments performed on the equimolar mixture of HAH1:MNK1.

Supplementary Table S4: Measured values of the correlation time $\left(\tau_{c}\right)$ for protein reorientation in solution for MNK1 and HAH1 isolated and in the HAH1-Cu(I)-MNK1 complex.

Supplementary Table S5: Statistical analysis of the solution structure of HAH1-Cu(I)-MNK1.

Supplementary Table S6: X-ray data collection and refinement statistics

Supplementary Table S7 Backbone RMSD values for HAH1 in the various structures available Supplementary Table S8 Backbone RMSD values for MNK1 in the various structures available. 


\section{Reference List}

1 Linder, M. C. (1991) Biochemistry of Copper, Plenum Press, New York

2 O'Halloran, T. V. and Culotta, V. C. (2000) Metallochaperones: An Intracellular Shuttle Service for Metal Ions. J.Biol.Chem. 275, 25057-25060

3 Harrison, M. D., Jones, C. E., Solioz, M., and Dameron, C. T. (2000) Intracellular copper routing: the role of copper chaperones. Trends Biochem.Sci. 25, 29-32

4 Puig, S. and Thiele, D. J. (2002) Molecular mechanisms of copper uptake and distribution. Curr.Opin.Chem.Biol. 6, 171-180

5 Banci, L., Bertini, I., Cantini, F., Felli, I. C., Gonnelli, L., Hadjiliadis, N., Pierattelli, R., Rosato, A., and Voulgaris, P. (2006) The Atx1-Ccc2 complex is a metal-mediated proteinprotein interaction. Nat.Chem.Biol. 2, 367-368

6 Yuan, D. S., Stearman, R., Dancis, A., Dunn, T., Beeler, T., and Klausner, R. D. (1995) The Menkes/Wilson disease gene homologue in yeast provides copper to a ceruloplasmin-like oxidase required for iron uptake. Proc.Natl.Acad.Sci.USA 92, 2632-2636

7 Lin, S. J., Pufahl, R., Dancis, A., O'Halloran, T. V., and Culotta, V. C. (1997) A role for the Saccharomyces cerevisiae ATX1 gene in copper trafficking and iron transport. J.Biol.Chem. 272, 9215-9220

8 Hamza, I., Schafer, M., Klomp, L. W., and Gitlin, J. D. (1999) Interaction of the copper chaperone HAH1 with the Wilson disease protein is essential for copper homeostasis. Proc.Natl.Acad.Sci.USA 96, 13363-13368

9 Banci, L., Bertini, I., Del Conte, R., D'Onofrio, M., and Rosato, A. (2004) Solution structure and backbone dynamics of the $\mathrm{Cu}(\mathrm{I})$ and apo-forms of the second metal-binding domain of the Menkes protein ATP7A. Biochemistry 43, 3396-3403

10 Anastassopoulou, J., Banci, L., Bertini, I., Cantini, F., Katsari, E., and Rosato, A. (2004) Solution structure of the apo-and copper(I) loaded human metallo-chaperone HAH1. Biochemistry 43, 13046-13053

11 Ishima, R. and Torchia, D. A. (2000) Protein dynamics from NMR. Nature Struct.Biol. 7, 740-743

12 Garrett, D. S., Seok, Y. J., Peterkofsky, A., Clore, G. M., and Gronenborn, A. M. (1997) Identification by NMR of the binding surface for the histidine-containing phosphocarrier protein HPr on the N-terminal domain of enzyme I of the escherichia coli phosphotransferase system. Biochemistry 36, 4393-4398

13 Sali, A. and Blundell, T. L. (1993) Comparative protein modelling by satisfaction of spatial restraints. J.Mol.Biol. 234, 779-815 
14 Dominguez, C., Boelens, R., and Bonvin, A. M. (2003) HADDOCK: a protein-protein docking approach based on biochemical or biophysical information. J.Am.Chem.Soc. 125, $1731-1737$

15 Banci, L., Bertini, I., Cantini, F., Chasapis, C., Hadjiliadis, N., and Rosato, A. (2005) A NMR study of the interaction of a three-domain construct of ATP7A with copper(I) and copper(I)HAH1: the interplay of domains. J.Biol.Chem. 280, 38259-38263

16 Banci, L., Bertini, I., Cantini, F., Della Malva, N., Migliardi, M., and Rosato, A. (2007) The different intermolecular interactions of the soluble copper-binding domains of the Menkes protein, ATP7A. J.Biol.Chem. 282, 23140-23146

17 Banci, L., Bertini, I., Cantini, F., Migliardi, M., Rosato, A., and Wang, S. (2005) An atomic level investigation of the disease-causing A629P mutant of the Menkes protein ATP7A. J.Mol.Biol. 352, 409-417

18 Banci, L., Bertini, I., Chasapis, C., Ciofi-Baffoni, S., Hadjiliadis, N., and Rosato, A. (2005) An NMR study of the interaction between the human copper(I) chaperone and the second and fifth metal-binding domains of the Menkes protein. FEBS J. 272, 865-871

19 Yatsunyk, L. A. and Rosenzweig, A. C. (2007) Copper(I) binding and transfer by the Nterminus of the Wilson disease protein. J.Biol.Chem. 282, 8622-8631

20 Oz, G. L., Pountney, D. L., and Armitage, I. M. (1998) NMR spectroscopy studies of I=1/2 metal ions in biological systems. Biochem.Cell.Biol. 76, 223-234

21 Tanchou, V., Gas, F., Urvoas, A., Cougouluegne, F., Ruat, S., Averseng, O., and Quemeneur, E. (2004) Copper-mediated homo-dimerisation for the HAH1 metallochaperone. Biochem.Biophys.Res.Commun. 325, 388-394

22 Wernimont, A. K., Huffman, D. L., Lamb, A. L., O'Halloran, T. V., and Rosenzweig, A. C. (2000) Structural basis for copper transfer by the metallochaperone for the Menkes/Wilson disease proteins. Nat Struct Biol 7, 766-771

23 DeSilva, T. M., Veglia, G., and Opella, S. J. (2005) Solution structures of the reduced and $\mathrm{Cu}(\mathrm{I})$ bound forms of the first metal binding sequence of ATP7A associated with Menkes disease. Proteins 61, 1038-1049

24 Bahadur, R. P., Chakrabarti, P., Rodier, F., and Janin, J. (2004) A dissection of specific and non-specific protein-protein interfaces. J.Mol.Biol. 336, 943-955

25 Lo Conte, L., Chothia, C., and Janin, J. (1999) The atomic structure of protein-protein recognition sites. J Mol Biol 285, 2177-2198

26 Arnesano, F., Banci, L., Bertini, I., Ciofi-Baffoni, S., Molteni, E., Huffman, D. L., and O'Halloran, T. V. (2002) Metallochaperones and metal transporting ATPases: a comparative analysis of sequences and structures. Genome Res. 12, 255-271

27 Hussain, F., Olson, J. S., and Wittung-Stafshede, P. (2008) Conserved residues modulate copper release in human copper chaperone Atox1. Proc.Natl.Acad.Sci.USA 105, 11158-11163 
28 Huffman, D. L. and O'Halloran, T. V. (2000) Energetics of Copper Trafficking Between the Atx1 Metallochaperone and the Intracellular Copper-transporter, Ccc2. J.Biol.Chem. 275, 18611-18614

29 Liu, T., Reyes-Caballero, H., Li, C., Scott, R. A., and Giedroc, D. P. (2007) Multiple metal binding domains enhance the $\mathrm{Zn}(\mathrm{II})$ selectivity of the divalent metal ion transporter AztA. Biochemistry 46, 11057-11068

30 Lutsenko, S., Barnes, N. L., Bartee, M. Y., and Dmitriev, O. Y. (2007) Function and regulation of human copper-transporting ATPases. Physiol Rev. 87, 1011-1046

31 Argüello, J. M., Eren, E., and Gonzalez-Guerrero, M. (2007) The structure and function of heavy metal transport P1B-ATPases. Biometals 20, 233-248

32 Hamza, I., Prohaska, J., and Gitlin, J. D. (2003) Essential role for Atox 1 in the coppermediated intracellular trafficking of the Menkes ATPase. Proc.Natl.Acad.Sci.USA 100, 12151220

33 Mandal, A. K. and Argüello, J. M. (2003) Functional roles of metal binding domains of the Archaeoglobus fulgidus Cu(+)-ATPase CopA. Biochemistry 42, 11040-11047

34 Saha, R. P., Bahadur, R. P., Pal, A., Mandal, S., and Chakrabarti, P. (2006) ProFace: a server for the analysis of the physicochemical features of protein-protein interfaces. BMC.Struct.Biol. 6, 11 


\section{Tables}

Table 1 Interprotein contacts in the present structures. For the NMR structure, short distances $(<$ $4.0 \AA$ A) occurring in $>50 \%$ of the family members are reported.

\begin{tabular}{|c|c|c|c|c|}
\hline \multicolumn{5}{|c|}{ HAH1-Cd(II)-MNK1 } \\
\hline \multicolumn{2}{|c|}{ HAH1 } & & \multicolumn{2}{c|}{ MNK1 } \\
\hline & & & & \\
\hline & & & & \\
\hline 12 & CYS & & 18 & CYS \\
\hline 14 & GLY & & 12 & GLY \\
\hline 14 & GLY & & 14 & THR \\
\hline & & & & \\
\hline 14 & GLY & & 18 & CYS \\
\hline & & & & \\
\hline 18 & ALA & & 65 & MET \\
\hline 18 & ALA & & 66 & GLY \\
\hline 18 & ALA & & 67 & PHE \\
\hline 21 & ARG+ & & 63 & ASP \\
\hline 21 & ARG+ & & 66 & GLY \\
\hline 21 & ARG+ & & 67 & PHE \\
\hline 21 & ARG+ & & 68 & ASP \\
\hline 22 & VAL & & 64 & ASP \\
\hline 22 & VAL & & 65 & MET \\
\hline 22 & VAL & & 66 & GLY \\
\hline 57 & LYS+ & & 25 & GLN \\
\hline 57 & LYS+ & & 64 & ASP \\
\hline 57 & LYS+ & & 65 & MET \\
\hline 58 & THR & & 21 & THR \\
\hline 58 & THR & & 65 & MET \\
\hline 59 & GLY & & 25 & GLN \\
\hline 60 & LYS+ & & 20 & TRP \\
\hline 60 & LYS+ & & 21 & THR \\
\hline & & & \\
\hline
\end{tabular}

\begin{tabular}{|c|c|c|c|c||}
\hline \multicolumn{5}{|c|}{ HAH1-Cu(I)-MNK1 } \\
\hline \multicolumn{2}{|c|}{ HAH1 } & & \multicolumn{2}{c|}{ MNK1 } \\
\hline 12 & CYS & & 15 & CYS \\
\hline 12 & CYS & & 17 & SER \\
\hline 12 & CYS & & 18 & CYS \\
\hline & & & & \\
\hline & & & & \\
\hline 14 & GLY & & 15 & CYS \\
\hline & & & & \\
\hline 15 & CYS & & 18 & CYS \\
\hline & & & & \\
\hline 18 & ALA & & 66 & GLY \\
\hline 18 & ALA & & 67 & PHE \\
\hline & & & & \\
\hline 21 & ARG+ & & 66 & GLY \\
\hline 21 & ARG+ & & 67 & PHE \\
\hline 21 & ARG+ & & 68 & ASP \\
\hline 22 & VAL & & 64 & ASP \\
\hline 22 & VAL & & 65 & MET \\
\hline & & & & \\
\hline & & & & \\
\hline 57 & LYS+ & & 64 & ASP \\
\hline & & & & \\
\hline 58 & THR & & 21 & THR \\
\hline 58 & THR & & 65 & MET \\
\hline & & & & \\
\hline 60 & LYS+ & & 20 & TRP \\
\hline 60 & LYS+ & & 21 & THR \\
\hline
\end{tabular}


Table 2. Properties of the protein-protein interface of various metal-mediated complexes (calculated on the experimental structures with the program Proface [34])

\begin{tabular}{|c|c|c|c|c|c|c|c|c|c|c|c|c|}
\hline & \multicolumn{3}{|c|}{ 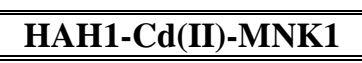 } & \multicolumn{3}{|c|}{ "HAH1-Cu(I)-MNK1 } & \multicolumn{3}{|c|}{ Yeast Atx-Cu(I)-Ccc2a } & \multicolumn{3}{|c|}{ HAH1 dimer } \\
\hline & HAH1 & MNK1 & Total & HAH1 & MNK1 & Total & Atx1 & Ccc2a & Total & HAH1 (Chain A) & HAH1 (Chain B) & Total \\
\hline Interface Area $\left(\AA^{2}\right)$ & 449 & 434 & 883 & 527 & 490 & 1017 & 416 & 426 & 842 & 494 & 478 & 972 \\
\hline $\begin{array}{c}\text { Interface Area / Surface } \\
\text { Area }\end{array}$ & 0.11 & 0.10 & 0.10 & 0.12 & 0.11 & 0.12 & 0.1 & 0.11 & 0.11 & 0.12 & 0.12 & 0.12 \\
\hline Number of atoms & 50 & 44 & 94 & 54 & 56 & 110 & 46 & 51 & 97 & 51 & 52 & 103 \\
\hline Number of residues & 15 & 14 & 29 & 16 & 14 & 30 & 15 & 15 & 30 & 15 & 15 & 30 \\
\hline $\begin{array}{l}\text { Fraction of non-polar } \\
\text { atoms }\end{array}$ & 0.70 & 0.64 & 0.67 & 0.61 & 0.54 & 0.57 & 0.59 & 0.49 & 0.54 & 0.59 & 0.58 & 0.58 \\
\hline $\begin{array}{c}\text { Non-polar interface area } \\
\left(\AA^{2}\right)\end{array}$ & 253 & 219 & 472 & 249 & 210 & 459 & 185 & 161 & 345 & 223 & 218 & 441 \\
\hline $\begin{array}{l}\text { Fraction of fully buried } \\
\text { atoms }\end{array}$ & 0.16 & 0.18 & 0.17 & 0.28 & 0.25 & 0.26 & 0.28 & 0.29 & 0.29 & 0.22 & 0.17 & 0.19 \\
\hline Residue Propensity Score & -1.87 & 0.46 & -1.41 & -2.18 & -0.21 & -2.39 & -2.32 & -0.17 & -2.49 & -1.61 & -1.67 & -3.28 \\
\hline Local Density & 32.76 & 28.95 & & 35.04 & 33.86 & & 29.91 & 33.53 & & 31.84 & 30.92 & \\
\hline
\end{tabular}


Table 3. Statistics for the structures of the adducts between each of the six MBDs of ATP7A and HAH1 calculated with the docking program HADDOCK. Only the best cluster of solutions was taken into account.

\begin{tabular}{|l|r|r|r|r|r|r||}
\hline & \multicolumn{1}{|c|}{ MNK1 } & \multicolumn{1}{c|}{ MNK2 } & \multicolumn{1}{c|}{ MNK3 } & \multicolumn{1}{c|}{ MNK4 } & \multicolumn{1}{c|}{ MNK5 } & \multicolumn{1}{c|}{ MNK6 } \\
\hline HADDOCK score & $-103.7 \pm 5.7$ & $-65.4 \pm 3.0$ & $-57.2 \pm 4.7$ & $-103.8 \pm 3.2$ & $-91.2 \pm 7.3$ & $-48.9 \pm 3.7$ \\
\hline RMSD from the overall lowest-energy structure $(\AA)$ & $1.2 \pm 0.9$ & $0.7 \pm 0.5$ & $1.2 \pm 0.7$ & $0.8 \pm 0.5$ & $0.6 \pm 0.4$ & $1.0 \pm 0.7$ \\
\hline Van der Waals Energy $\left(\mathrm{kcal} \mathrm{mol}^{-1}\right)$ & $-28.1 \pm 6.8$ & $-36.1 \pm 2.8$ & $-36.4 \pm 3.5$ & $-27.7 \pm 0.8$ & $-46.9 \pm 5.5$ & $-26.7 \pm 2.0$ \\
\hline Electrostatic Energy $\left(\mathrm{kcal} \mathrm{mol}^{-1}\right)$ & $-391 \pm 43$ & $-242 \pm 17$ & $-89 \pm 13$ & $-382 \pm 12$ & $-206 \pm 20$ & $-246 \pm 20$ \\
\hline Desolvation Energy $\left(\mathrm{kcal} \mathrm{mol}^{-1}\right)$ & $1.3 \pm 4.1$ & $18.4 \pm 3.5$ & $-3.6 \pm 3.3$ & $-0.7 \pm 0.9$ & $-6.6 \pm 2.3$ & $26.3 \pm 6.3$ \\
\hline Restraints violation Energy $\left(\mathrm{kcal} \mathrm{mol}^{-1}\right)$ & $13 \pm 11$ & $7.4 \pm 3.6$ & $5.4 \pm 3.5$ & $9.6 \pm 2.5$ & $35 \pm 27$ & $7.1 \pm 2.6$ \\
\hline
\end{tabular}




\section{Figure Legends}

Figure 1. Comparison of the solution structures of A) HAH1-Cu(I)-MNK1; B) Cu(I)-HAH1 (PDB code: 1TL4); C) $\mathrm{Cu}(\mathrm{I})-\mathrm{MNK} 1$ (PDB code: $1 \mathrm{KVJ}$ ). The side chains of the metal-binding cysteines are shown in green. The fourth cysteine, which does not bind the metal in the present structure, is shown in grey.

Figure 2 Crystal structure of HAH1-Cd(II)-MNK1 (same orientation as in Figure 1A). The side chains of the metal-binding cysteines are shown in green; the side chain of metal-binding threonine is in dark green. The fourth cysteine is shown in grey.

Figure 3 Electrostatic potential surfaces of left) MNK1 and right) HAH1 in the HAH1-Cu(I)MNK1. The backbone and side chains (as of Table 1) of the partner are also shown.

Figure 4. Combined chemical shift variations of selected residues for WT-HAH1 and the three interface mutants. Not all peaks could be detected in the Thr58Ala mutant. 


\section{B. Biochemical Journal Immediate Publication. Published on 19 May 2009 as manuscript BJ20090422}

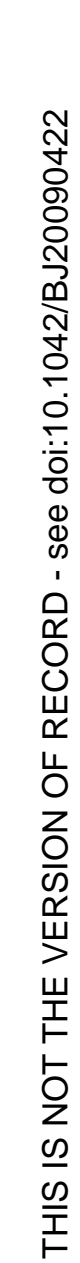

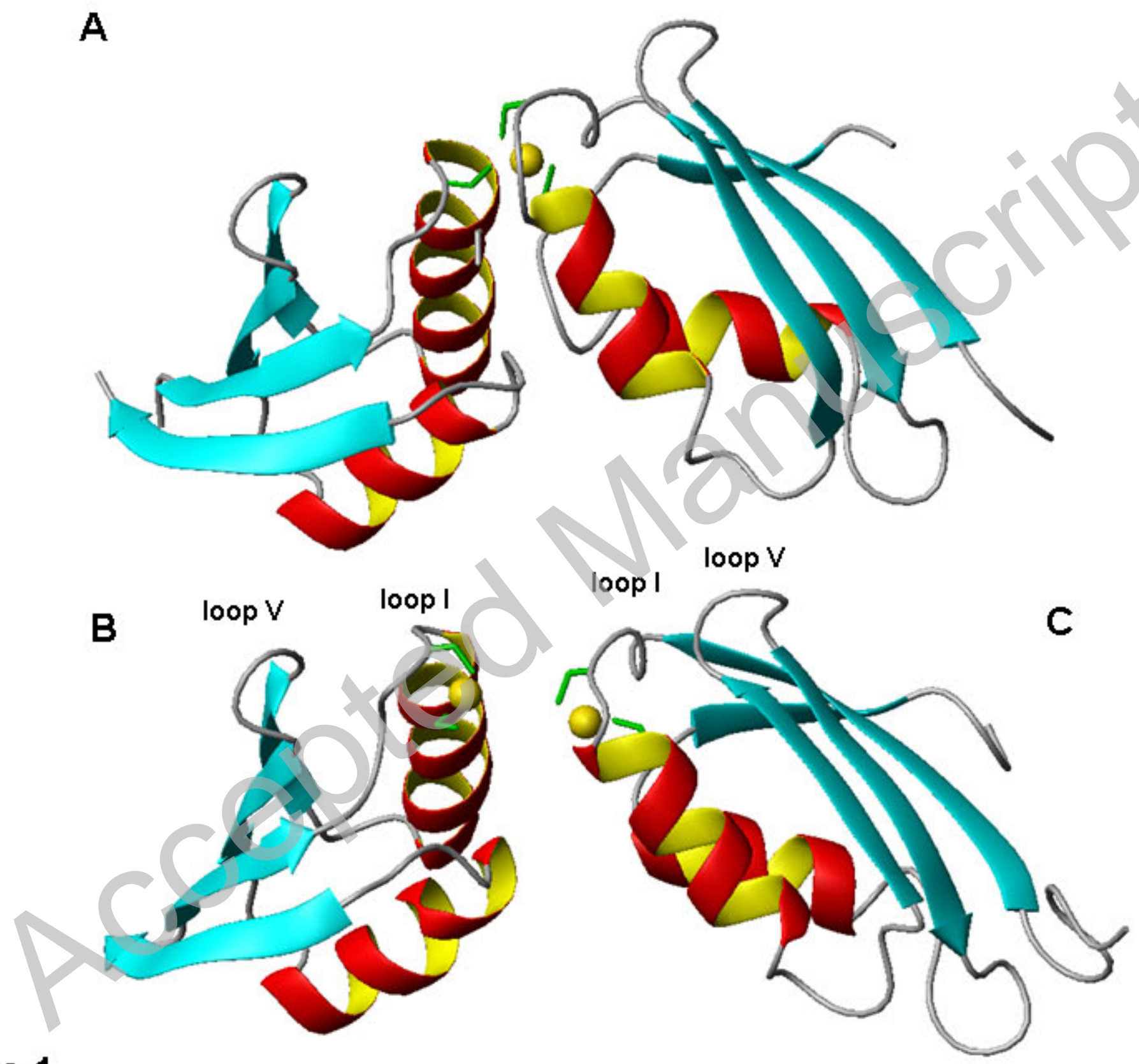

Figure 1.

Licenced copy. Copying is not permitted, except with prior permission and as allowed by law.

(C) 2009 The Authors Journal compilation (c 2009 Portland Press Limited 


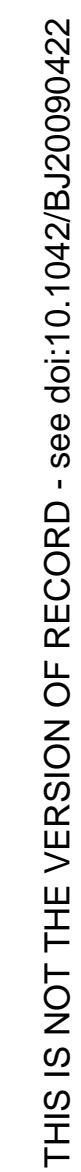

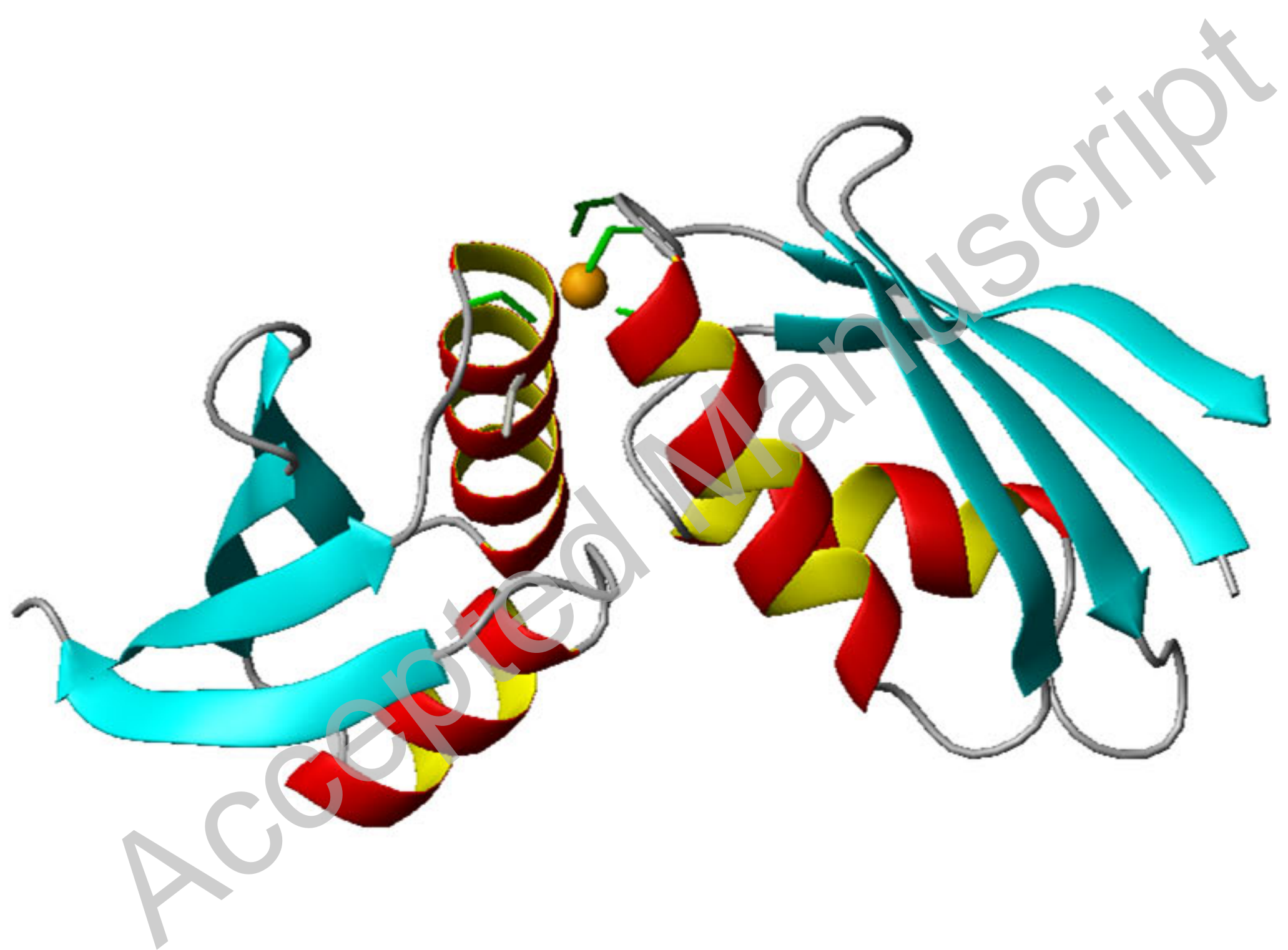

Figure 2.

Licenced copy. Copying is not permitted, except with prior permission and as allowed by law. (C) 2009 The Authors Journal compilation (C 2009 Portland Press Limited 

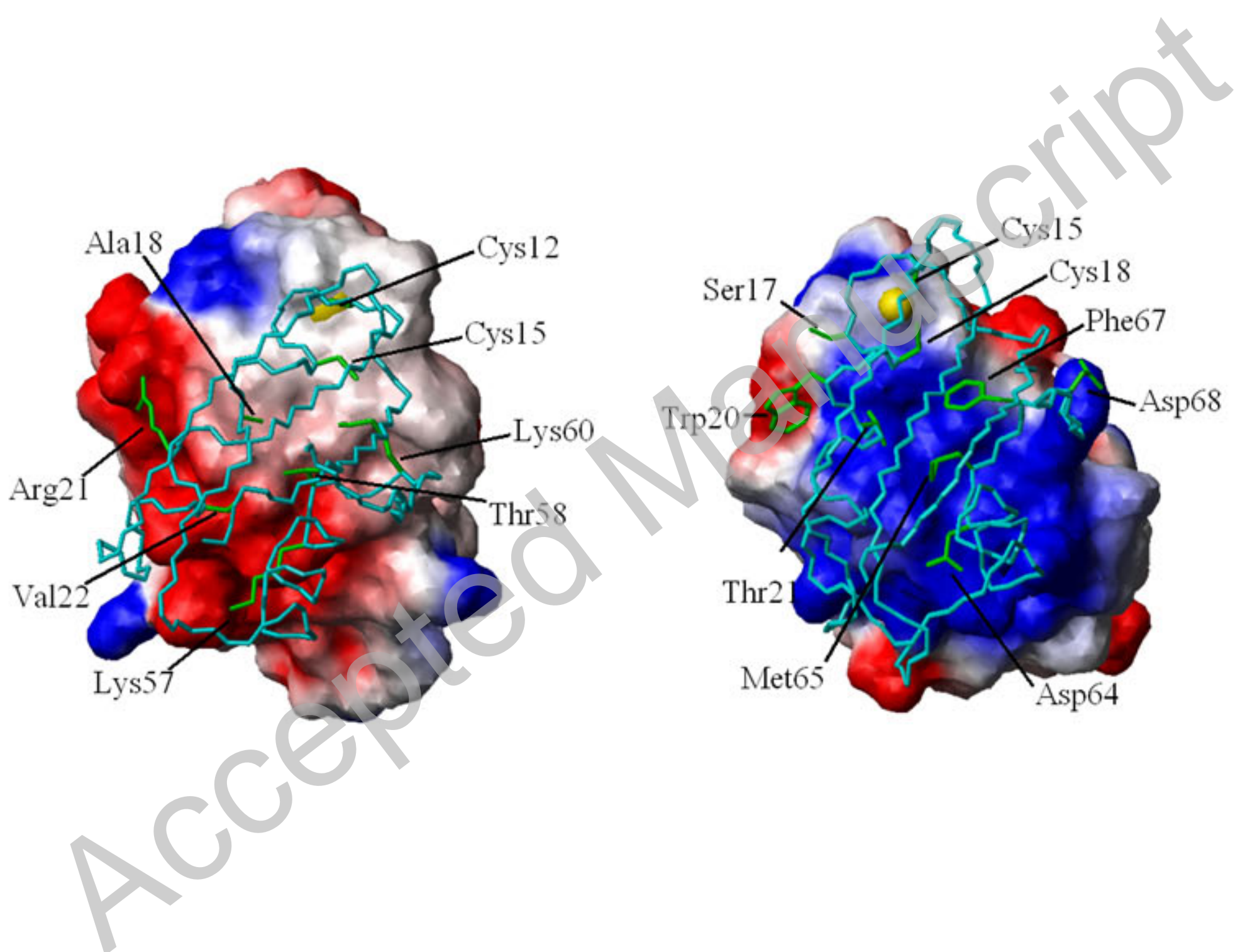

Figure 3.

Licenced copy. Copying is not permitted, except with prior permission and as allowed by law.

(C) 2009 The Authors Journal compilation (C) 2009 Portland Press Limited 


\section{B Biochemical Journal Immediate Publication. Published on 19 May 2009 as manuscript BJ20090422}

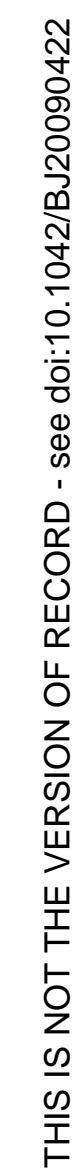

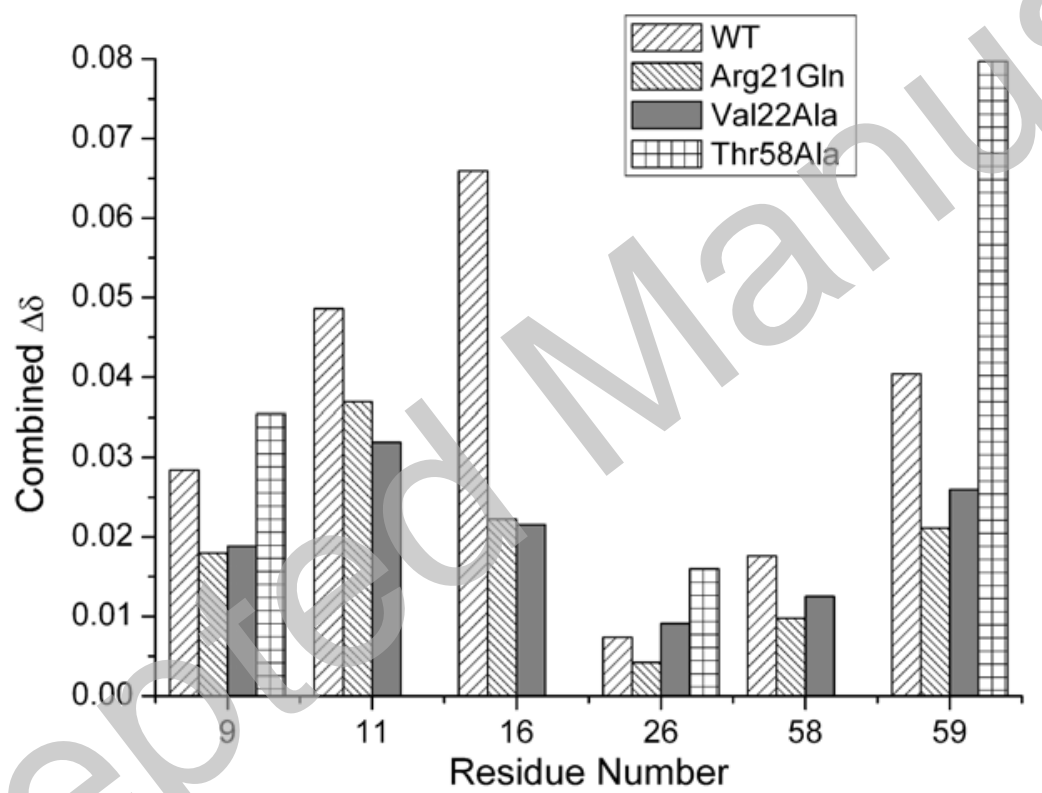

Figure 4.

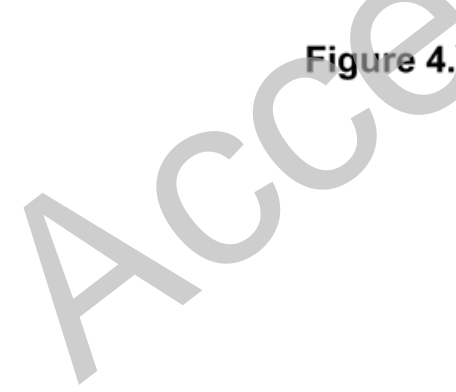

Licenced copy. Copying is not permitted, except with prior permission and as allowed by law. (C) 2009 The Authors Journal compilation (C) 2009 Portland Press Limited 